\title{
Mapping quantitative trait loci for five forage quality traits in a sorghum-sudangrass hybrid
}

\author{
J.Q. Li*, L.H. Wang*, Q.W. Zhan, Y.L. Liu, Q. Zhang, J.F. Li and F.F. Fan \\ College of Agriculture, Anhui Science and Technology University, Fengyang, China \\ *These authors contributed equally to this study. \\ Corresponding author: Q.W. Zhan \\ E-mail: qwzhan@163.com
}

Genet. Mol. Res. 14 (4): 13266-13273 (2015)

Received May 7, 2015

Accepted August 19, 2015

Published October 26, 2015

DOI http://dx.doi.org/10.4238/2015.October.26.23

\begin{abstract}
The identification of quantitative trait loci (QTLs) affecting forage quality traits enables an understanding of the genetic mechanism of these loci. The aim of the present study was to detect QTLs for the wholeplant protein content (WP), whole-plant fat content (WF), neutral detergent fiber (NDF), acid detergent fiber (ADF), and whole-plant ash content (WA) using a population of $184 \mathrm{~F}_{2}$ individuals from a cross between sorghum Tx623A and sudangrass Sa. Correlation analysis was performed between the five forage quality traits. WP was found to be positively correlated with WF, NDF, and ADF. Furthermore, NDF was positively correlated with ADF but negatively correlated with WA. A genetic map with 124 SSR markers was constructed for QTL mapping. A total of 12 QTLs associated with the five forage quality traits were detected. Of these QTLs, qNDF3, qNDF8, and qADF8 explained more than $10 \%$ of the phenotypic variation. Additionally, although all of the QTLs exhibited additive and dominant effects, they mainly exhibited dominant effects. Our results provide important information for marker-assisted selection breeding of sorghum-sudangrass hybrids.
\end{abstract}

Key words: ADF; Forage quality traits; NDF; Quantitative trait loci; Sorghum-sudangrass hybrid 


\section{INTRODUCTION}

Sorghum (Sorghum bicolor L.) is a multi-usage crop that can be used as a food source, for animal feed, and as raw material for industries. Sudangrass (Sorghum sudanese L.) is an annual forage crop with a high tiller and yield. Both sorghum and sudangrass are diploids with $2 \mathrm{n}=20$ and belong to the same genus. Compared to their parents, sorghum-sudangrass hybrids have a high forage yield and nutritive value. These hybrids exhibit prominent inter-specific heterosis and are regarded as high-quality forage for cattle and sheep (Zhan and Qian, 2004; Beck et al., 2007). In recent years, these advantages have led to an increase in the area of cultivated sorghumsudangrass hybrids in China.

Breeding sorghum-sudangrass hybrids for high quality forage traits is an important goal because it contributes to animal meat and milk production. Whole-plant protein, fat, fiber, ash, and fiber contents are important quality traits for forage. The fiber content can be quantified as neutral detergent fiber (NDF) and acid detergent fiber (ADF). Most crop research has found that these traits are controlled by quantitative trait loci (QTLs) (Cardinal et al., 2003; Cogan et al., 2005; Liu et al., 2012). Therefore, dissection of these QTLs will be helpful for the breeding of forage quality in sorghum-sudangrass hybrids.

With the development of molecular markers, high-density molecular genetic maps for QTLs have subsequently been developed for crops. In addition, QTLs controlling forage quality traits have also been identified in ryegrass, maize, barley, and rice straw (Cogan et al., 2005; Grandoet al., 2005; Xie et al., 2011; Courtial et al., 2013). In contrast, there have been no reports of gene mapping of forage quality traits in sorghum-sudangrass hybrids. The main objectives of this research were to characterize the inheritance pattern and identify the controlling locioffive forage quality traits using an $F_{2}$ population. The results will provide information for the improvement of forage quality in sorghum-sudangrass hybrids.

\section{MATERIAL AND METHODS}

\section{Mapping population}

A set of $184 \mathrm{~F}_{2}$ plants was gathered from a cross between Tx623A (sorghum) and Sa (sudangrass). Sorghum Tx623A was donated by Professor F.R. Miller, while Sudangrass Sa is a local variety in Anhui Province, China (Zhan et al., 2006). The $F_{2}$ plants and their parents were sown in the experimental field of Anhui Science and Technology University with a planting density of $50 \times 25 \mathrm{~cm} . \mathrm{F}_{2}$ plants and 20 parental plants were sown in the same field, with 10 plants per row. Standard agronomic practices were applied from sowing to harvest.

\section{Phenotype measurements}

$F_{2}$ individuals and 10 parent plants were hand-harvested with 15-cm stubble when both of the parents were at heading (more than $50 \%$ of the plants were at heading). After harvest, the samples were dried in a forced-air oven for $48 \mathrm{~h}$ at $60^{\circ} \mathrm{C}$. The samples were ground with a mill and scanned through a reflectance-based diode array analyzer (Perten DA7200, Sweden). Analyzed traits included the whole-plant protein content (WP), whole-plant fat content (WF), NDF, ADF, and whole-plant ash (WA) by selecting the forage quality measurement in the machine. 


\section{DNA extraction and simple sequence repeat (SSR) marker analysis}

The leaves of parent and $F_{2}$ plants were sampled and used for genomic DNA extraction. DNA was extracted using the sodium dodecyl sulfate (SDS) method as previously described (Murray and Thompson 1980). A total of 924 SSR markers were selected from 10 sorghum chromosomes and screened for polymorphisms between the parents of the $F_{2}$ population. These markers included 257 that encoded AH (Lu et al., 2009), 371 that encoded GS (Ramuet al., 2010), 196 that encoded TXP (Wu and Huang, 2007), and 100 that encoded Y (Zhan et al. 2008). The polymorphic SSR markers were then used to genotype the $F_{2}$ individuals. Information relating to the SSR markers used in the study is shown in a supplemental file (Table S1).

Polymerase chain reaction (PCR) was performed using a $10 \mu \mathrm{L} \mathrm{PCR} \mathrm{mixture} \mathrm{composed}$ of $2 \mu \mathrm{L}$ DNA $(20 \mathrm{ng} / \mu \mathrm{L}), 1 \mu \mathrm{L} 10 \mathrm{X}$ buffer $\left(20 \mathrm{mM} \mathrm{Mg}^{2+}\right), 1.5 \mu \mathrm{L}$ primers $(2 \mathrm{pmol} / \mu \mathrm{L}), 1.0 \mu \mathrm{LdNTPs}$ (1 mM), $0.5 \mu \mathrm{LrTaq}$ DNA polymerase $(2 \mathrm{U} / \mu \mathrm{L})$, and $4 \mu \mathrm{L}$ deionized-distilled $\mathrm{H}_{2} \mathrm{O}$. The samples were pre-denatured at $94^{\circ} \mathrm{C}$ for $5 \mathrm{~min}$, followed by 36 cycles of $30 \mathrm{~s}$ at $94^{\circ} \mathrm{C}, 30 \mathrm{~s}$ at $55^{\circ} \mathrm{C}$, and $80 \mathrm{~s}$ at $72^{\circ} \mathrm{C}$, and a final extension at $72^{\circ} \mathrm{C}$ for $7 \mathrm{~min}$. PCR products were detected using silver staining on an $8 \%$ non-denaturing polyacrylamide gel.

\section{Data analysis, linkage map construction, and QTL analysis}

To characterize the relationship between phenotypic traits, a correlation analysis was performed. Pearson correlation coefficients were calculated using DPS 7.0 for Windows (Tang and Zhang, 2013). Mapping data were analyzed using JoinMap 4.0. The linkage groups were determined from the results of pair-wise comparisons at a minimum likelihood of odds (LOD) value of 2-10. The best order was determined by comparing the goodness-of-fit of the resulting map for each tested order using a threshold of 1.0 and 2.0 for the linkage groups and loci, respectively. The Kosambi map function was used to calculate the genetic distances.

QTLs were detected using Windows QTL IciMapping version 4.0 (Wang et al., 2014). Inclusive Composite Interval Mapping (ICIM) was performed to identify the putative QTLs (Li et al., 2008a). A threshold of LOD > 2.5 was used to declarethe presence of putative QTL. The QTLs were detected using a 1.0-cM (CentiMorgan) step in scanning.

\section{RESULTS}

\section{Construction of the genetic linkage map}

Agenetic map was constructed from $184 \mathrm{~F}_{2}$ individuals and consisted of 124 SSR markers. Ten linkage groups were formed with an average distance of $10.4 \mathrm{cM}$ between the markers. The longest distance between the markers was $17.7 \mathrm{cM}$, and the shortest distance between the markers was $1.8 \mathrm{cM}$. The details of the linkage map are shown in Figure 1.

\section{Statistical analysis of the phenotype data}

The phenotypic trait means and range of the $\mathrm{F}_{2}$ individuals along with two parents for five traits are presented in Table 1. The two parental lines varied significantly in their mean performance for all of the traits. Values for WP, WF, NDF, and ADF in the parental line Tx623A were higher than those in the other parental line, Sa, and the WA of Tx623A was significantly lower than that 
of Sa. Therefore, the forage quality of Tx623A was better than that of Sa in terms of the five traits analyzed.

Among the $F_{2}$ population, wide segregation was found in the five traits. The trait means of the $F_{2}$ population were intermediate between the two parental lines, and transgressive segregations were observed for all traits in the population. The kurtosis and skewness for WP, WF, NDF, and ADF were less than 1, indicating their normal distribution (Table 1). The kurtosis of WA was greater than 1 , indicating that it displayed a relatively peaked distribution.
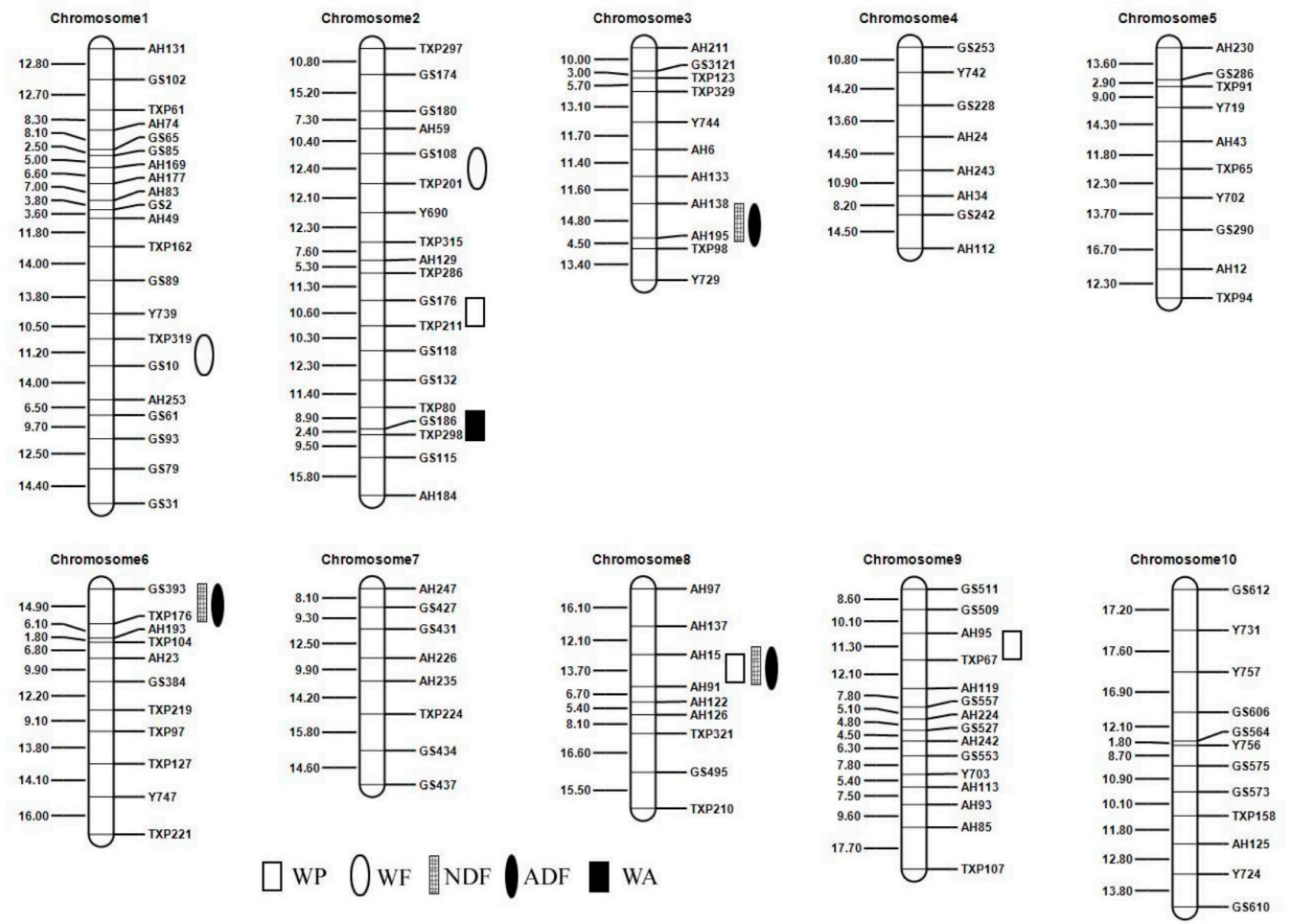

Figure 1. Linkage map of the Tx623A x Sa $F_{2}$ population with the positions of QTLs shown on the right side of the chromosomes. WP: whole-plant protein content, WF: whole-plant fat content, NDF: neutral detergent fiber, ADF: acid detergent fiber, WA: whole-plant ash content.

\section{Table 1. Variation in the five forage quality traits of the parents and the $F_{2}$ population.}

\begin{tabular}{llccccc}
\hline Name & Parameter & WP $(\%)$ & WF $(\%)$ & NDF $(\%)$ & ADF $(\%)$ & WA (\%) \\
\hline Tx623A & Mean & 11.3 & 3.32 & 60.2 & 38.1 & 6.43 \\
Sa & Mean & 7.46 & 2.31 & 54.5 & 32.6 & 8.45 \\
$\mathrm{~F}_{2}$ & Mean & 8.42 & 3.31 & 61.18 & 36.8 & 7.58 \\
& Range & $6.11-13.04$ & $2.82-3.97$ & $55.41-67.06$ & $31.5-42.83$ & $4.14-8.86$ \\
& SD & 2.14 & 0.21 & 2.08 & 2.05 & 0.61 \\
& Kurtosis & 0.08 & 0.91 & 0.06 & 0.05 & 2.21 \\
& Skewness & -0.24 & 0.66 & 0.37 & 0.49 & -0.78 \\
\hline
\end{tabular}

WP: whole-plant protein content, WF: whole-plant fat content, NDF: neutral detergent fiber, ADF: acid detergent fiber, WA: whole-plant ash content. 


\section{Correlation of the traits}

Pearson correlation coefficients among the five measured traits were estimated and are listed in Table 2. WP was positively correlated with WF, NDF, and ADF; the correlations were highest with NDF (0.92), followed by ADF (0.91), and WA (0.48). NDF was positively correlated with ADF but negatively correlated with WA. ADF was also negatively correlated with WA. In addition, WF was not correlated with NDF, ADF, or WA.

Table 2. Correlation analysis among five forage quality traits of the $F_{2}$ population.
\begin{tabular}{lcccc}
\hline Traits & Correlation coefficient & & ADF (\%) \\
\cline { 2 - 5 } & WP $(\%)$ & 1.0 & NDF $(\%)$ & \\
\hline WP & 1.0 & 0.34 & 1.0 & 1.0 \\
WF & $0.48^{*}$ & 0.38 & $0.97^{* *}$ & $-0.5^{*}$ \\
NDF & $0.92^{* *}$ & -0.62 & $-0.47^{*}$ & 1.0 \\
ADF & $0.91^{* *}$ & -0.42 & WA
\end{tabular}

*Indicates significance at the 0.05 level, ${ }^{* *}$ Indicates significance at the 0.01 level.

\section{QTL analysis for five forage quality traits}

Phenotypic and genotypic data for $184 \mathrm{~F}_{2}$ individuals were subject to QTL analysis. The putative QTLs for each trait in the population identified by the ICIM are listed in Table 3. The chromosomal location of QTLs is depicted in Figure 1. A total of 12 putative QTLs that were significantly associated with the five forage quality traits were identified. These QTLs were distributed on six chromosomes, including chromosomes 1, 2, 3, 6, 8, and 9.

\begin{tabular}{|c|c|c|c|c|c|c|c|}
\hline Trait & QTL & Chromosome & Marker & LOD & Additive & Dominance & $\mathrm{R} 2$ \\
\hline \multirow[t]{3}{*}{ WP } & $q W P 2$ & 2 & GS176-TXP211 & 2.75 & -0.2087 & -1.3654 & 5.42 \\
\hline & qWP8 & 8 & AH15-AH91 & 3.10 & 0.2825 & 1.8824 & 9.05 \\
\hline & $q W P 9$ & 9 & АH95-TXP67 & 2.77 & 0.0043 & 1.6194 & 6.54 \\
\hline \multirow[t]{2}{*}{ WF } & $q W F 1$ & 1 & TXP319-GS10 & 3.63 & -0.0618 & 0.1179 & 7.61 \\
\hline & $q W F 2$ & 2 & GS108-TXP201 & 3.11 & 0.1003 & 0.0591 & 9.41 \\
\hline \multirow[t]{3}{*}{ NDF } & qNDF3 & 3 & $\mathrm{AH} 138-\mathrm{AH} 195$ & 2.83 & -0.1631 & 1.8651 & 11.31 \\
\hline & qNDF6 & 6 & GS393-TXP176 & 3.06 & -0.4581 & 1.2851 & 5.75 \\
\hline & qNDF8 & 8 & AH15-AH91 & 2.92 & -0.0291 & 2.1341 & 11.37 \\
\hline \multirow[t]{3}{*}{ ADF } & $q A D F 3$ & 3 & $\mathrm{AH} 138-\mathrm{AH} 195$ & 2.92 & -0.45 & 1.9470 & 6.61 \\
\hline & qADF6 & 6 & GS393-TXP176 & 3.32 & -0.4508 & 1.3623 & 6.08 \\
\hline & $q A D F 8$ & 8 & AH15-AH91 & 2.81 & -0.0313 & 2.2729 & 12.82 \\
\hline WA & gWA2 & 2 & GS186-TXP29 & 3.08 & -0.2520 & -0.1233 & 8.33 \\
\hline
\end{tabular}

\section{QTLs for the whole-plant protein content}

Three QTLs were detected for the whole-plant protein content. These QTLs were located on chromosomes 2, 5, and 9. The phenotypic variation explained by each QTL ranged from 5.42 to $9.05 \%$, and the LOD scores ranged from 2.75 to 3.10 . The Tx623A allele of $q W P 2$ decreased the whole-plant protein content, whereas the Tx632A alleles of the other two QTLs increased the whole-plant protein content. The absolute value of additive effects for the three QTLs was greater 
than the absolute value of dominant effects, indicating that dominant effects play a more important role in controlling the whole-plant protein content.

\section{QTLs for the whole-plant fat content}

Two QTLs located on chromosomes 1 and 2 were found for the whole-plant fat content. The phenotypic variation in $q W F 1$ and $q W F 2$ was 7.61 and $9.41 \%$, as detected with LOD of 3.63 and 3.11. The Tx623A allele of $q W F 1$ increased the whole-plant fat content, but the Tx623A allele of $q W F 2$ decreased the whole-plant fat content. qWF1 had mainly dominant effects, while qWF2 had mainly additive effects.

\section{QTLs for NDF and ADF}

Three QTLs that co-located on the same region of three different chromosomes were detected for NDF and ADF. Two major QTLs, qNDF3 and qNDF8, which were identified on chromosomes 3 and 8 , explained 11.3 and $11.7 \%$, respectively. A major QTL, $9 A D F 8$, which was identified on chromosome 8 , explained $12.82 \%$. The additive effects of these QTLs were negative; the dominant effects were positive. The absolute value of the additive effects of these QTLs was smaller than the absolute value of the dominant effects, indicating that dominant effects played a more important role in these two traits.

\section{QTL for the whole-plant ash content}

For the trait of whole-plant ash content (WA), only one QTL was detected. qWA2 was located at the interval GS186-TXP29 on chromosome 2 and was detected with an LOD of 3.08. This QTL explained $8.33 \%$ of the phenotypic variation. The Tx623A allele of $q W A 2$ decreased the WA. The absolute value of the additive effects was greater than that of the dominant effects, indicating that $q W A 2$ mainly had additive effects.

\section{DISCUSSION}

Following the development of molecular genetics, an increasing number of high-density linkage maps have been constructed using different marker systems and populations (Mace and Jordan, 2010, Alamet al., 2014). However, relatively few studies have investigated sorghumsudangrass hybrids and, to date, only one linkage map has been constructed using random amplification polymorphic DNA (RAPD) and amplified fragment length polymorphism (AFLP) markers of these hybrids (Lu et al., 2011). Compared to RAPD and AFLP markers, SSR markers are highly polymorphic and co-dominant. We constructed the first linkage maps in these hybrids using full SSR markers. AH series markers were developed by our research team based on the expressed sequence tag (EST) sequence of sorghum (Li et al., 2010). These markers are reliable for use in molecular genetic research investigating sorghum-sudangrass hybrids.

Heterosis represents one of the most revolutionary advances in crop improvement (Lippman and Zamir, 2007). The genetic basis of heterosis has been debated for more than 100 years and has still not been completely resolved (Stuber et al., 1992; Frascaroli et al., 2007). Additive QTL do not contribute to heterosis, in contrast to non-additive QTL, including dominant, overdominant, and epistatic QTL (Lu et al., 2003; LeDeauxet al., 2006; Li et al., 2008b; He et al., 
2012). The elite variety WanCao No. 3, which is a hybrid of Tx623A and Sa, had great heterosis in yield and quality traits (Zhan et al., 2006). We located nine QTLs associated with forage quality traits. The absolute value of the dominant effects was greater than the absolute value of the additive effects in eight QTLs, suggesting that dominant effects might be the primary genetic basis of heterosis in forage quality traits of sorghum-sudangrass hybrids.

NDF and ADF are important indicators of forage quality because of their negative relationship with digestibility for livestock animals (Krakowsky et al., 2006). ADF consists largely of cellulose and lignin; NDF consists largely of cellulose, hemicelluloses, and lignin. We noted three QTLs associated with NDF and ADF that were co-located on the same region of the same chromosome. The difference between NDF and ADF is hemicellulose. There may be fewer differences in semi-fiber content between Tx623A and Sa. The co-location of NDF and ADF has also been observed in rice straw (Xie et al., 2011). Additionally, qNDF8, qADF8, and qWA8 were co-localized on the same region of chromosome 8 . We also found that WF was positively correlated with NDF and ADF, which could explain whyqNDF8, $q A D F 8$, and $q W A 8$ were co-localized to the same region. In addition, we could select the favorable alleles of the QTL for quality breeding in sorghum-sudangrass hybrids because this QTL controls the three traits.

According to the relative positions of the markers on the reference sorghum genome (www.gramene.org), a search was performed for candidate genes involved in cellulose and lignin biosynthesis (Li et al., 2013). A gene that is similar to glycosyltransferase (Sb03g026550) in conjugating lignin monomers was found close to qADF3 and qNDF3 on chromosome 3 . One putative cell wall component (Sb06g033580) was located at the region of qADF6 and qNDF6. However, whether the QTLs were true genes that are involved in lignin or cellulose biosynthesis requires further fine mapping of these traits.

\section{Conflicts of interest}

The authors have no conflict of interest.

\section{ACKNOWLEDGMENTS}

Research supported by the National Natural Science Foundation (\#31301383), the Keyconstruction Subject Plan of Anhui Province (\#WanJiaoMiKe[2014]28), the Special Program of Scientific Research from China Agriculture Department (\#201503133), the Crop Science Keyconstruction subject plan of Anhui Science and Technology University (\#AKZDXK2015A03).

\section{Supplementary material}

\section{REFERENCES}

Alam MM, Mace ES, van OosteromEJ, Cruickshank A, et al. (2014). QTL analysis in multiple sorghum populations facilitates the dissection of the genetic and physiological control of tillering. Theor. Appl. Genet. 127: 2253-2266.

Beck PA, Hutchison S, Gunter SA, Losi TC, et al. (2007). Chemical composition and in situ dry matter and fiber disappearance of sorghum x Sudangrass hybrids. J. Anim. Sci. 85: 545-55.

Cardinal AJ, Lee M and Moore KJ (2003). Genetic mapping and analysis of quantitative trait loci affecting fiber and lignin content in maize. Theor. Appl. Genet. 106: 866-874.

Cogan NO, Smith KF, Yamada T, Francki MG, et al. (2005). QTL analysis and comparative genomics of herbage quality traits in perennial ryegrass (Lolium perenne L.). Theor. Appl. Genet. 110: 364-380. 
Courtial A, Thomas J, Reymond M, Méchin V, et al. (2013). Targeted linkage map densification to improve cell wall related QTL detection and interpretation in maize. Theor. Appl. Genet. 126: 1151-1165.

Frascaroli E, Canè MA, Landi P, Pea G, et al. (2007). Classical genetic and quantitative trait loci analyses of heterosis in a maize hybrid between two elite inbred lines. Genetics 176: 625-644.

Grando S, Baum M, Ceccarelli S, Goodchild A, et al. (2005). QTLs for straw quality characteristics identified in recombinant inbred lines of a Hordeumvulgare $\mathrm{x}$. spontaneum cross in a Mediterranean environment. Theor. Appl. Genet. 110: 688-695.

He XH, Hu ZL and Zhang YM (2012). Genome-wide mapping of QTL associated with heterosis in the RIL-based NCIII design. Chin. Sci. Bull. 21: 2655-2665.

Krakowsky MD, LeeM and Coors JG (2006). Quantitative trait loci for cell wall components in recombinant inbred lines of maize (Zea mays L.) II: leaf sheath tissue. Theor. Appl. Genet. 112: 717-726.

Le Deaux JR, Graham GI and Stuber CW (2006). Stability of QTLs involved in heterosis in maize when mapped under several stress conditions. Maydica 51: 151-167.

Li H, Ribaut JM, Li Z and Wang J (2008a). Inclusive Composite interval mapping (ICIM) for digenic epistasis of quantitative traits in biparental populations. Theor. Appl. Genet. 116: 243-260.

Li L, Lu K, Chen Z, Mu T, et al. (2008b). Dominance, overdominance and epistasis condition the heterosis in two heterotic rice hybrids. Genetics 180: 1725-1742.

Li J, Wang L, Zhan Q, Liu Y, et al. (2013). Sorghum bmr6 mutant analysis demonstrates that a shared MYB1 transcription factor binding site in the promoter links the expression of genes in related pathways. Funct. Integr. Genomics 13: 445-453

Li JQ, Wang LH, Zhan QW and Chen Y (2010). Establishment of sorghum EST-SSR marker and its preliminary application to sudangrass. Prat. Sci. 27: 112-117.

Lippman ZB and Zamir D (2007). Heterosis: revisiting the magic. Trends Genet. 23: 60-66.

Liu L, Stein A, Wittkop B, Sarvari P, et al. (2012). A knockout mutation in the lignin biosynthesis gene CCR1 explains a major QTL for acid detergent lignin content in Brassica napus seeds. Theor. Appl. Genet. 124: 1573-1586.

Lu H, Romero-Severson J and Bernardo R (2003). Genetic basis of heterosis explored by simple sequence repeat markers in a random-mated maize population. Theor. Appl. Genet. 107: 494-502.

Lu J, Lv YY, Li JQ, Zhan QW, et al. (2009). SSR primer designing and construction of a genetic map of Sorghum bicolor $x$ S. sudanense. Chin. J. Grass. 31: 28-33.

Lu XP, Yun JF, Gao CP and Acharya S (2011). Quantitative trait loci analysis of economically important traits in Sorghum bicolor x S. sudanense hybrid. Can. J. Plant Sci. 91: 81-90.

Mace ES and Jordan DR (2010). Location of major effect genes in sorghum (Sorghum bicolor (L.) Moench). Theor. Appl. Genet. 121: 1339-1356.

Murray MG and Thompson WF (1980). Rapid isolation of high molecular weight plant DNA. Nucleic Acids Res. 8: 4321-4325.

Ramu P, Desphande SP, Senthilvel S, Jayashree B, et al. (2010). In silico mapping of important genes and markers available in the public domain for efficient sorghum breeding. Mol. Breed. 26: 409-418.

Stuber CW, Lincoln SE, Wolff DW, Helentjaris T, et al. (1992). Identification of genetic factors contributing to heterosis in a hybrid from two elite maize inbred lines using molecular markers. Genetics 132: 823-839.

Tang QY and Zhang CX (2013). Data Processing System (DPS) software with experimental design, statistical analysis and data mining developed for use in entomological research. Insect Sci. 20: 254-260.

Wang JK, Li HH, Zhang Y and Meng L (2014). Users' Manual of QTL IciMapping v4.0. Institute of Crop Science, CAAS, Beijing

Wu YQ and Huang Y (2007). An SSR genetic map of Sorghum bicolor (L.) Moench and its comparison to a published genetic map. Genome 50: 84-89.

Xie JK, Kong XL, Chen J, Hu BL, et al. (2011). Mapping of quantitative trait loci for fiber and lignin contents from an interspecific cross Oryza sativaxOryzarufipogon. Zhejiang Univ-Sci B (Biomed. \& Biotechnol.) 12: 518-526.

Zhan QW, Lin P and Qian ZQ (2006). Breeding and characteristic of Wancao No 3 sorghum-sudangrass hybrid. Crops 4: 35-36.

Zhan QW and Qian ZQ (2004). Heterosis utilization of hybrid between sorghum [Sorghum bicolor (L.) Moench] and sudangrass [Sorghum sudanense (Piper) Stapf]. Acta. Agron. Sin. 30: 73-77.

Zhan QW, Zhang TZ, Wang BH and Li JQ (2008). Diversity comparison and phylogenetic relationships of S. bicolor and S. sudanenseas revealed by SSR markers. Plant Sci. 174: 9-16. 